\title{
Role of autophagy on bone marrow mesenchymal stem-cell proliferation and differentiation into neurons
}

\author{
BO $\mathrm{LI}^{1}$, PING DUAN ${ }^{1}$, CAIFANG LI $^{2,3}$, YING JING $^{1}$, XUEFEI HAN ${ }^{3}$, WENHAI YAN ${ }^{2,3}$ and YING XING ${ }^{1,3}$ \\ Departments of ${ }^{1}$ Physiology and ${ }^{2}$ Pathophysiology, Zhengzhou University, Zhengzhou, Henan 450001; \\ ${ }^{3}$ Stem Cell Research Center of Basic Medicine College, Zhengzhou University, Zhengzhou, Henan 450052, P.R. China
}

Received January 19, 2015; Accepted October 28, 2015

DOI: $10.3892 / \mathrm{mmr} .2015 .4673$

\begin{abstract}
The purpose of the present study was to investigate the role of autophagy on rat bone marrow mesenchymal stem cell (BMSC) proliferation, apoptosis and differentiation into neurons. After treatment with rapamycin, 3-methyladenine (3-MA) or chloroquine, the cell cycle, apoptosis, expression of neuron-specific enolase (NSE) and the mean fluorescence intensity (MFI) of Notch1 in BMSCs were examined by flow cytometry. The expression of microtubule-associated protein 2 (MAP2), Notch1 and Hes1 was investigated by western blot analysis. The results showed that after induction of autophagy using rapamycin, the proliferation of BMSCs was inhibited. Furthermore, the S-phase population was significantly decreased compared to that in the control group $(\mathrm{P}<0.05)$. In addition, the percentage of NSE-positive cells and the expression of MAP2 were significantly increased compared to those in the control group $(\mathrm{P}<0.05)$. The MFI of Notch1 was markedly upregulated compared to that in the control group $(\mathrm{P}<0.05)$. When autophagy was inhibited by 3-MA or chloroquine, the percentage of apoptotic cells and NSE-positive cells as well as the expression of MAP2 were markedly reduced compared to those in the control group $(\mathrm{P}<0.05)$. Furthermore, western blot analysis showed that Notch1 and Hes1 were decreased in the rapamycin-treated group, while they were not affected by 3-MA or chloroquine. The present study indicated that induction of autophagy in BMSCs decreased their S-phase population, promoted their differentiation into neurons and promoted the expression of NSE and MAP2. The mechanisms
\end{abstract}

Correspondence to: Professor Wenhai Yan, Department of Pathophysiology, Zhengzhou University, 100 Science Avenue, High-Tech Zone, Zhengzhou, Henan 450001, P.R. China

E-mail: yanwh@zzu.edu.cn

Professor Ying Xing, Department of Physiology, Zhengzhou University, 100 Science Avenue, High-Tech Zone, Zhengzhou, Henan 450001, P.R. China

E-mail: xingy@zzu.edu.cn

Key words: bone marrow mesenchymal stem cells, autophagy, Notch1, proliferation, differentiation underlying this process may be linked to the regulation of autophagy-induced inhibition of the Notch1 signaling pathway.

\section{Introduction}

Autophagy is a conserved and important metabolic pathway in eukaryotic cells. Under starvation or stress conditions, harmful and unnecessary intracellular macromolecules are degraded and recycled through autophagy, which thereby promotes cell survival and growth (1). In populations of undifferentiated cells as well as cells undergoing self-renewal or differentiation into mature cells, the cells exhibit protein renewal and organelle degradation (2). Autophagy, which is stimulated by the macroenvironment or cytokines, has an important role in remodeling stem cells, thus regulating cell differentiation and self-renewal. However, the role of autophagy in bone marrow-derived mesenchymal stem cell (BMSC) proliferation and differentiation has remained elusive.

Notch1 signaling is a regulator which is widely distributed in various organs of developing embryos (3), a variety of adult organs as well as undifferentiated cells with the ability to proliferate, and which has important roles in the development, proliferation and differentiation of various cell types (4-6). Notch1 signaling has been shown to have important roles in BMSC proliferation and differentiation, where it exerts an activating and inhibitory function, respectively (7). Furthermore, induction of autophagy is associated with the Notch1 signaling pathway. In order to investigate the role of autophagy in BMSC proliferation and differentiation, as well as the underlying molecular mechanisms, the present study used autophagy inducer rapamycin and autophagy inhibitors 3-methyladenine (3-MA) and chloroquine, to modulate autophagic activity in BMSCs. The effects of the modified autophagic activity on BMSC proliferation, apoptosis and differentiation into neurons were examined, and the role of the Notch1 signaling pathway in this process was investigated.

\section{Materials and methods}

Animals. Adult male Sprague-Dawley rats $(n=30$; age, four weeks, weight, 160-200 g) were obtained from the Animal Center of Zhengzhou University (Zhengzhou, China) and housed in a specific pathogen-free room. Animal procedures were approved by the Ethics Committee of Zhengzhou 
University (Zhengzhou, China) and performed according to the 'Guidance On the Care and Use of Laboratory Animals' (People's Republic of China National Science and Technology Committee; 2006). BCMCs were obtained three times from 10 rats.

Reagents. The reagents used in the present study were as follows: $0.25 \%$ Trypsin-EDTA (1X), Dulbecco's modified Eagle's medium (DMEM), fetal bovine serum (FBS) (all from Gibco; Thermo Fisher Scientific, Waltham, MA, USA), radioimmunoprecipitation assay (RIPA) lysis buffer, phenylmethanesulfonyl fluoride (PMSF), bicinchoninic acid (BCA) protein assay kit (cat. no. P0010s), and Electro-Chemi-Luminescence Plus kit (cat. no. P0018; all from Beyotime Institute of Biotechnology, Haimen, China), Tris, glycine and SDS (all from Beijing Solarbio Science \& Technology Co., Ltd., Beijing, China), Temed (Shanghai Huashuo Fine Chemical Co., Ltd., Shanghai, China), ammonium persulfate (Tianjin Kemiou Chemical Reagent Co., Ltd., Tianjin, China), rapamycin, 3-MA, chloroquine and dimethylsulfoxide (DMSO) (all from Sigma-Aldrich, St. Louis, MO, USA), rabbit polyclonal anti-Notch1, rabbit polyclonal anti-Hes1, rabbit monoclonal anti-P62, and rabbit polyclonal anti-microtubule-associated protein 2 (MAP2) antibodies (All from Abcam, Cambridge, MA, USA; cat. nos. ab33932, ab49170, ab109012, and ab32454; 1:1,000), rabbit-anti polyclonal microtubule-associated protein 1 light chain $3 \mathrm{~B}$ (LC3B), and rabbit-anti polyclonal $\beta$-actin antibodies (Cell Signaling Technology, Inc., Danvers, MA, USA; cat. nos. 3868 and $4967 ; 1: 1,000$ ), and horseradish peroxidase-labeled goat anti-rabbit secondary antibodies (Santa Cruz Biotechnology, Inc.; cat. no. SC-2004; 1:2,000).

Isolation, purification and induction of BMSCs. Following sacrificing the rats by cervical dislocation, bone marrow cells were collected by repeatedly flushing both femurs and tibias with culture medium using a $5-\mathrm{ml}$ syringe under the sterile/cell culture hood. Cells at $1 \times 10^{6} / \mathrm{ml}$ were seeded into culture dishes $\left(75 \mathrm{~cm}^{2}\right)$ and cultured in a humidified incubator containing 5\% $\mathrm{CO}_{2}$ at $37^{\circ} \mathrm{C}$, and the medium was changed every 3-4 days. 6-7 days later, cells were re-seeded at the ratio of 1:2.

Cells in the third passage were randomly divided into four groups: The control group and three groups treated with rapamycin $(10 \mathrm{mmol} / \mathrm{l}), 3-\mathrm{MA}(3 \mathrm{mmol} / \mathrm{l})$ and chloroquine $(25 \mathrm{mmol} / \mathrm{l})$, respectively, for $12 \mathrm{~h}$. Experiments were performed in triplicate. For the experiments on cell differentiation, cells were divided into the same groups as mentioned above, but cultured with induction medium [low-glucose DMEM (Gibco; Thermo Fisher Scientific, Inc.) containing 2\% DMSO and $200 \mu \mathrm{mol} / 1$ butylated Hydroxyanisole (BHA; Sigma-Aldrich)] for $12 \mathrm{~h}(8)$.

\section{Flow cytometry}

Cell cycle analysis. After trypsinization and washing in phosphate-buffered saline (PBS) twice, cells were re-suspended with $200 \mu \mathrm{l}$ cold PBS and fixed with $800 \mu \mathrm{l}$ chilled ethanol, which was added drop wise with agitation. The suspensions were stored at $-20^{\circ} \mathrm{C}$ overnight, followed by centrifugation (179 x g, $5 \mathrm{~min}$, room temperature), re-suspension of the cell pellet in propidium iodide (PI; BD Biosciences,
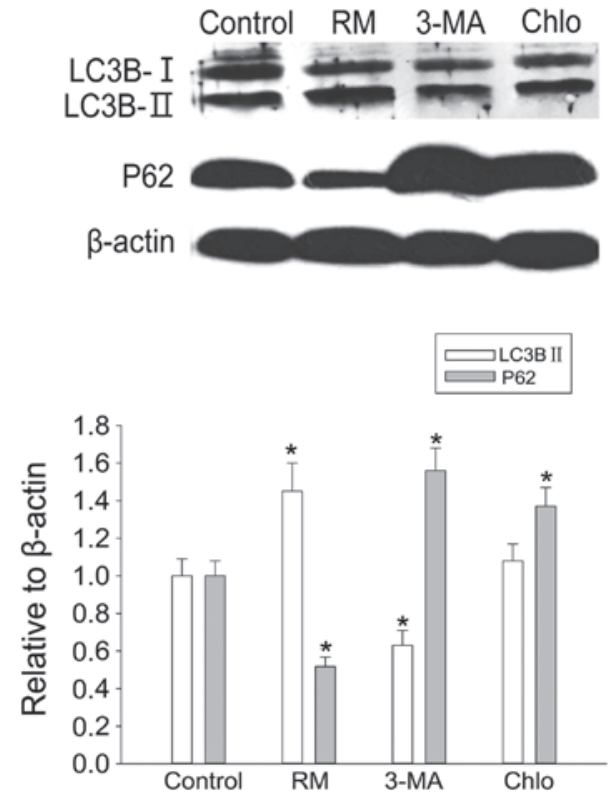

Figure 1. Effects of various drugs on autophagic activity of bone marrow mesenchymal stem cells following incubation for $12 \mathrm{~h}$. Autophagy inducer RM promoted the expression of LC3B-II and inhibited the expression of P62. Autophagy inhibitors 3-MA and chloroquine increased the expression of P62. Treatment with 3-MA reduced LC3B-II expression, while chloroquine slightly promoted LC3B-II expression. * $\mathrm{P}<0.05$ vs. control group. RM, rapamycin; Chlo, chloroquine; LC3B, microtubule-associated protein 1 light chain $3 \mathrm{~B}$; 3-MA, 3-methyladenine.

Erembodegem, Belgium)/RNase staining buffer (200 $\mu \mathrm{l}$; BD Biosciences, Franklin Lakes, NJ, USA) and incubation in the dark for $15 \mathrm{~min}$ at room temperature. The cell cycle was then analyzed by flow cytometry.

Assessment of apoptosis. Cell treatments were conducted according to the protocol of the fluorescein isothiocyanate (FITC)-Annexin V Apoptosis Detection kit (BD Biosciences; cat. no. 556547). After trypsinization and washing in PBS twice, cells were re-suspended in $100 \mu 11 \mathrm{X}$ Annexin V binding buffer, $5 \mu 1$ FITC-Annexin V and $5 \mu \mathrm{l}$ PI (all from BD Biosciences), and incubated in the dark for 15 min after gentle mixing. Following addition of $200 \mu \mathrm{l} 1 \mathrm{X}$ Annexin V binding buffer, apoptosis was analyzed by flow cytometry (BD FACSCanto II; BD Biosciences) within $1 \mathrm{~h}$.

Analysis of neuron-specific markers neuron-specific enolase (NSE) and Notch1. Cells were fixed and permeabilized using the BD cytofix/cytoperm kit (cat. no. 554714; BD Biosciences, Franklin Lakes, NJ, USA) according to the manufacturer's instructions. In brief, $500 \mu 1$ Fixation/Permeabilization solution was added and cells were incubated in the dark for $20 \mathrm{~min}$ at room temperature. After centrifugation (179 x g, $5 \mathrm{~min}$, room temperature), cell pellets were re-suspended in $2 \mathrm{ml}$ BD Perm/wash buffer and incubated in the dark for $10 \mathrm{~min}$ at room temperature. Subsequently, phycoerythrin (PE)-conjugated mouse IgG anti-Notch1 antibodies ( $1 \mu \mathrm{l}$; BD Biosciences; cat. no. 552768; 1:100) or rabbit polyclonal anti-NSE (Abcam; cat. no. ab53025; 1:500) were added, followed by incubation for $30 \mathrm{~min}$ at room temperature in the dark or $2 \mathrm{~h}$ on ice in the dark, respectively. After the cells were washed using BD Perm/wash buffer, the cells incubated with anti-Notch1 antibodies were re-suspended 
A

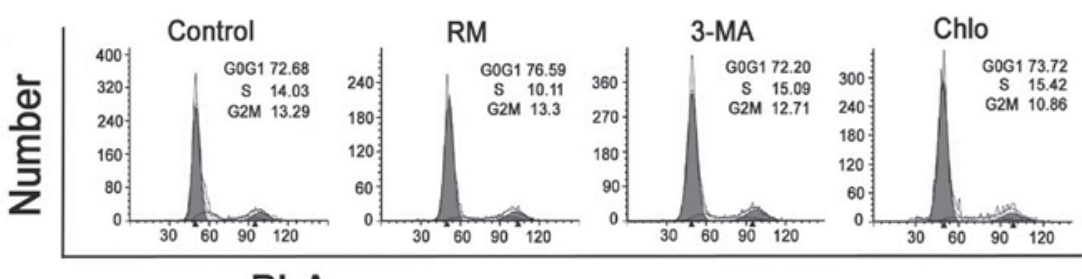

$\mathrm{PI}-\mathrm{A}$

B

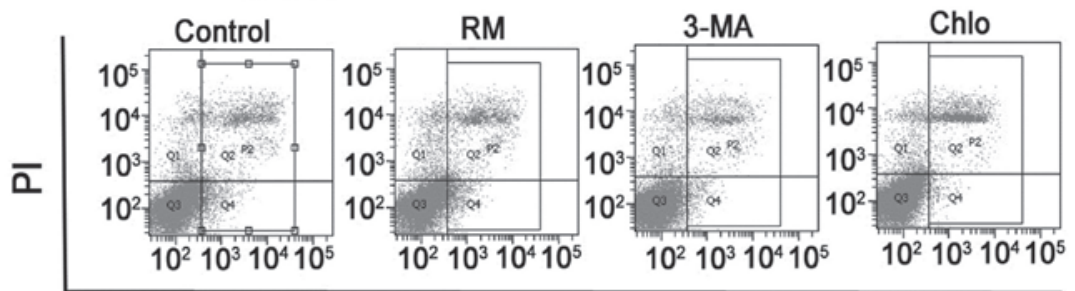

Annexin V FITC

C

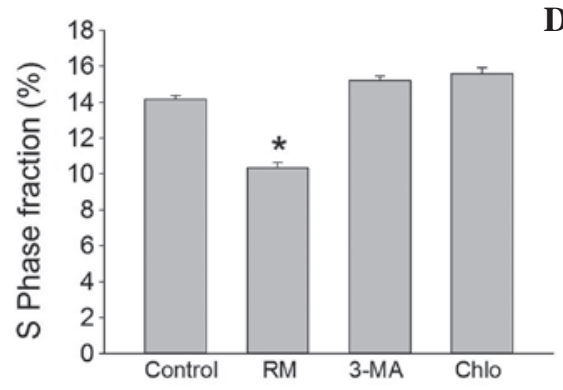

D

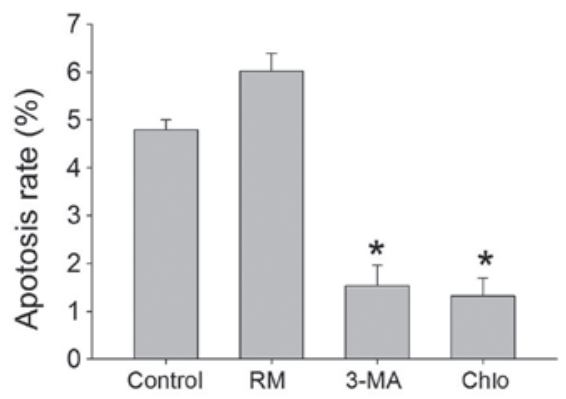

Figure 2. Effects of autophagy on cell cycle and apoptosis of BMSCs. After incubation of BMSCs with various drugs for $12 \mathrm{~h}$, (A) cell cycle and (B) apoptosis were detected by flow cytometry. (C) The S-phase population in the control, RM-treated, 3-MA-treated and Chlo-treated groups was 14.03, 10.11, 15.09 and $15.42 \%$, respectively. (D) The percentage of apoptotic cells in the control, RM-treated, 3-MA-treated and Chlo-treated groups was 4.7, 5.9, 1.5 and 1.3\%, respectively. Values are expressed as the mean \pm standard deviation. "P $<0.05$ vs. control group. RM, rapamycin; Chlo, chloroquine. BMSC, bone marrow mesenchymal stem cell; 3-MA, 3-methyladenine; PI, propidium iodide; FITC, fluorescein isothiocyanate.

in $300 \mu \mathrm{l}$ PBS containing $1 \%$ paraformaldehyde and stored at $4^{\circ} \mathrm{C}$, whereas those incubated with anti-NSE antibodies were treated with PE-conjugated rabbit IgG antibodies (BD Biosciences; cat. no. 558416; 1:1,000) for $1 \mathrm{~h}$ on ice in the dark. The cells were then washed and re-suspended as above. The ratio of NSE-positive cells and the mean fluorescence intensity (MFI) of Notch1 were analyzed using flow cytometry (BD FACSCanto II) with a Data-Interpolating Vibrational Analysis software version 5.0 (BD Biosciences) for data acquisition and analysis.

Western blot analysis. Following cell lysis and protein extraction using RIPA lysis buffer, the protein concentration was measured using a BCA assay according to the instructions of the BCA kit. Protein in loading buffer $(40 \mu \mathrm{g})$ was resolved by $10 \%$ sodium dodecyl polyacrylamide electrophoresis (using 80-V constant-voltage electrophoresis until the bromophenol blue entered the gel, followed by $120-\mathrm{V}$ constant-voltage electrophoresis for 90 min; Beijing Dingguo Changsheng Biotechnology Co., Ltd., Beijing, China) and transferred onto a polyvinylidene difluoride membrane $(250 \mathrm{~mA}$ for $1 \mathrm{~h}$; Beijing Dingguo Changsheng Biotechnology Co., Ltd.). After blocking with 5\% skimmed milk for $1 \mathrm{~h}$ at room temperature, membranes were incubated with rabbit-anti LC3B, rabbit-anti MAP2, rabbit-anti P62, rabbit anti-Notch1, rabbit anti-Hes1 $(1: 1,000)$ or $\beta$-actin antibodies $(1: 2,000)$ overnight at $4^{\circ} \mathrm{C}$.
After washing with Tris-buffered saline containing Tween 20 three times, membranes were then incubated with horseradish peroxidase-conjugated goat anti-rabbit antibody $(1: 2,000)$ for $1 \mathrm{~h}$ at room temperature. The membranes were further washed three times for $5 \mathrm{~min}$. Bands were detected using the Electro-Chemi-Luminescence Plus kit. Grey value analysis was performed using Quantity One software version 4.6.2 (Bio-Rad Laboratories, Inc., Hercules, CA, USA). $\beta$-actin was used as a loading control, and the expression levels of the proteins were normalized to $\beta$-actin.

Statistical analysis. Values are expressed as the mean \pm standard deviation. Statistical analysis was performed using the Student's t-test with SPSS 13.0 software (SPSS, Inc., Chicago, IL, USA). $\mathrm{P}<0.05$ was considered to indicate a significant difference between values.

\section{Results}

Pharmacological modulation of autophagic activity in $B M S C s$. Treatment with autophagy inducer rapamycin for $12 \mathrm{~h}$ promoted the expression of LC3B-II and reduced P62 expression in BMSCs, while autophagy inhibitors 3-MA and chloroquine produced opposite effects, except for a slight enhancement of LC3B-II expression following chloroquine treatment (Fig. 1). These results indicated that rapamycin 
A

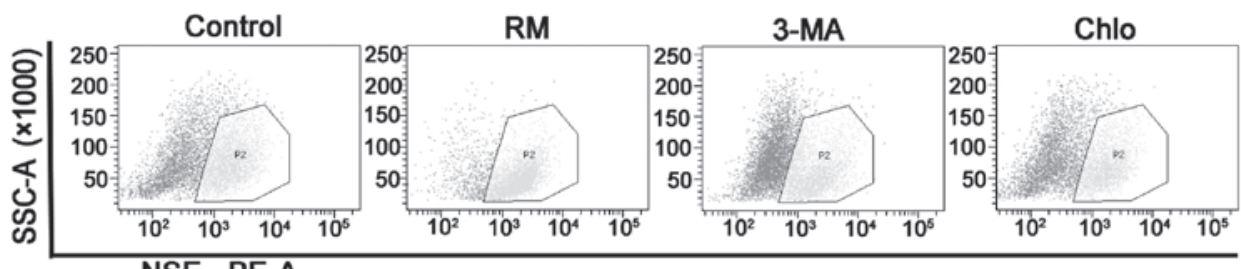

B

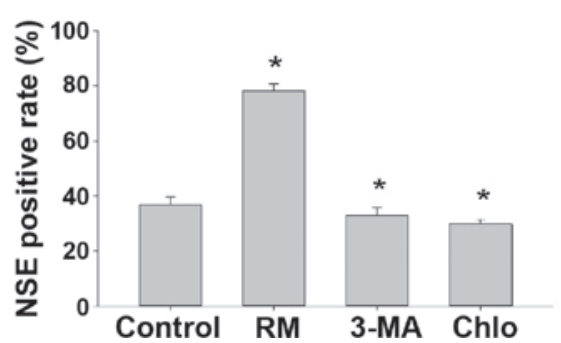

D
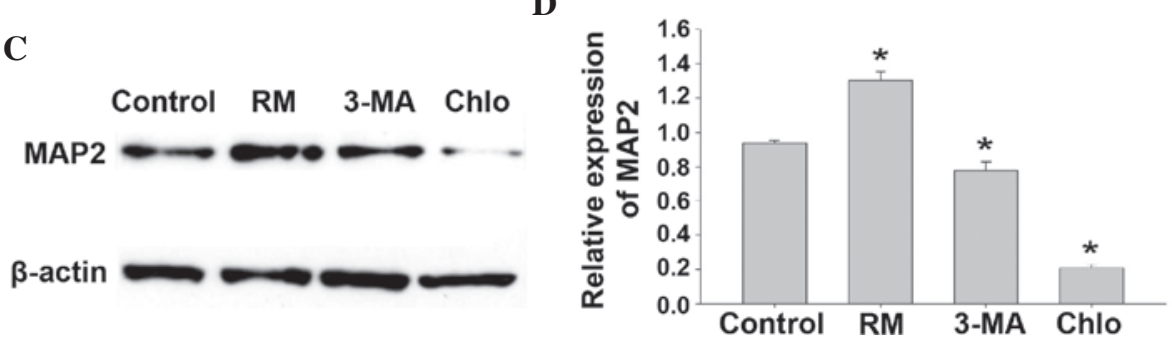

Figure 3. Effects of autophagy on the differentiation of BMSCs into neurons. (A and B) Following induction of BMSCs to differentiate into neurons for $12 \mathrm{~h}$, flow cytometric analysis revealed that the percentage of NSE-positive cells in the control, RM-treated, 3-MA-treated and Chlo-treated groups was 38.2, 80.1, 33.7 and 29.7\%, respectively. (C and D) Western blot analysis showed that RM treatment promoted the expression of MAP2, while treatment with 3-MA or Chlo inhibited MAP2 expression. Values are expressed as the mean \pm standard deviation. * $\mathrm{P}<0.05$ vs. control group. RM, rapamycin; Chlo, chloroquine; MA, methyladenine; BMSC, bone marrow mesenchymal stem cell; SSC, side scatter; MAP2, microtubule-associated protein 2; NSE, neuron-specific enolase; PE-A; absorption of phycoerythrin.

A

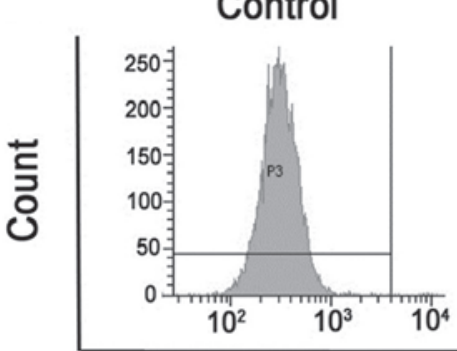

RM

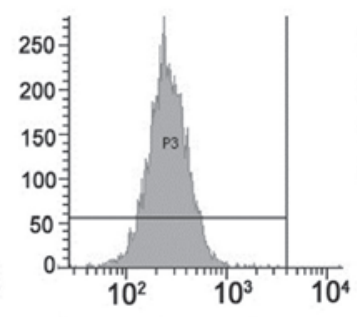

3-MA

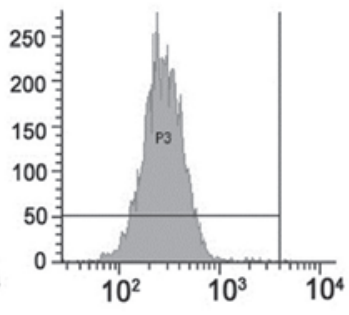

Chlo

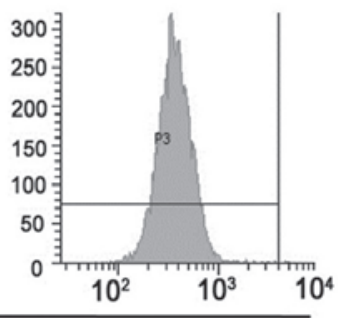

B

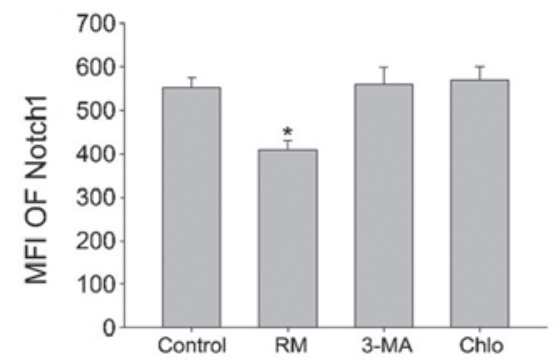

C
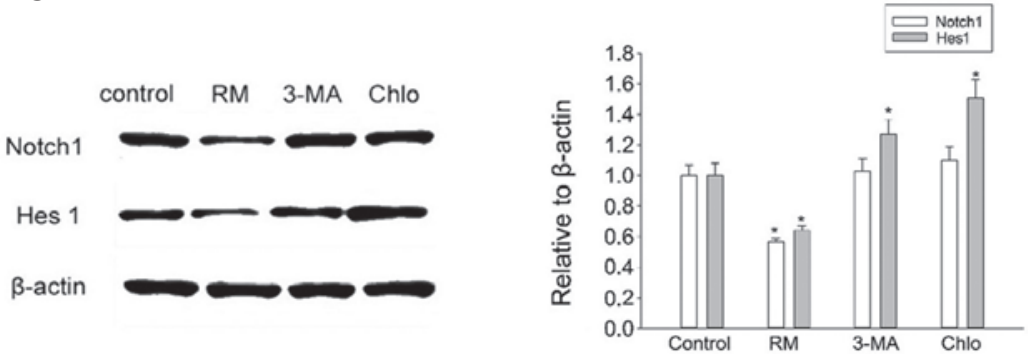

Figure 4. Effects of autophagy on Notch1 signaling in BMSCs. (A and B) Following incubation of BMSCs with various drugs for $12 \mathrm{~h}$, flow cytometric analysis revealed that the MFI of Notch1 was 562,415, 587 and 582 in the control, RM-treated, 3-MA-treated and Chlo-treated group, respectively. Values are expressed as the mean \pm standard deviation. ${ }^{*} \mathrm{P}<0.05$ vs. control group. (C) Western blot analysis showed that RM treatment reduced the expression of Notch1 and Hes1, while treatment with 3-MA or Chlo did not affect the expression of Notch1 and Hes1. RM, rapamycin; Chol, chloroquine; MFI, mean fluorescence intensity; MA, methyladenine; BMSC, bone marrow mesenchymal stem cell; PE-A; absorption of phycoerythrin.

was able to enhance autophagy, while 3-MA and chloroquine inhibited autophagy in BMSCs.

Autophagy inhibits the cell cycle of BMSCs. Cell cycle analysis BMSCs showed that induction of autophagy by rapamycin inhibited the cell proliferation, as indicated by a reduced S-phase cell population $(11.04 \pm 0.86 \%)$ when compared to that in the control group $(14.18 \pm 0.76 \% ; \mathrm{P}<0.05)$ (Fig. 2). Conversely, treatment with 3-MA and chloroquine marginally increased the S-phase cell population to $15.13 \pm 0.17 \%$ and $15.56 \pm 0.29 \%$, respectively; however, differences from the control group were not statistically significant (Fig. 2).

Autophagy promotes BMSC apoptosis. Flow cytometric analysis showed that autophagy inducer rapamycin slightly enhanced the apoptotic rate of BMSCs $(5.8 \pm 0.42 \%)$, which was, however, not significantly different from that of the control group $(4.6 \pm 0.55 \%)$. Following treatment with autophagy inhibitors 3-MA or chloroquine, the apoptotic rate of BMSCs $(1.5 \pm 0.1$ and $1.23 \pm 0.25$, respectively) was significantly reduced 
when compared to that in the control group $(\mathrm{P}<0.05)$ (Fig. 2). These results indicated that autophagy promotes apoptosis, while inhibition of autophagy prevents apoptosis of BMSCs.

Autophagy enhances the differentiation of BMSCs into neurons. BMSCs at passage 3 were spindle-shaped and had a fibroblast-like morphology. Following incubation in induction medium (low-glucose DMEM with $2 \%$ DMSO and $200 \mu \mathrm{mol} / 1$ BHA) for $12 \mathrm{~h}$, cells exhibited neurite extensions and their shape was changed to resemble neurons. Flow-cytometric analysis showed that rapamycin-induced autophagy promoted BMSC differentiation into neurons, as demonstrated by a significantly increased number of NSE-positive cells $(78.2 \pm 2.6)$ compared to that in the control group $(36.7 \pm 2.8 ; \mathrm{P}<0.05)$ (Fig. $3 \mathrm{~A}$ and $\mathrm{B})$. Conversely, treatment with autophagy inhibitors 3-MA or chloroquine reduced the percentage of NSE-positive cells (32.8 \pm 3.1 and $29.9 \pm 1.56$, respectively) compared that in the control group $(\mathrm{P}<0.05)$ (Fig. 3A and B). Furthermore, western blot analysis showed that upon differentiation into neurons, the expression of microtubule-associated protein 2 (MAP2) was increased in the rapamycin-treated and reduced in the 3-MAor chloroquine-treated groups compared to that in the control group $(\mathrm{P}<0.05$ for all) (Fig. 3C and D).

The effect of autophagy on Notch1 signaling pathway in $B M S C s$. To investigate the role of the Notch1 signaling pathway in the observed autophagy-mediated enhancement of neuronal differentiation of BMSCs, flow cytometry was used to detect Notch1 MFI after modulation of autophagy. The results showed that the Notch1 MFI was significantly reduced after treatment with rapamycin $(410 \pm 19)$, when compared to that in the control group $(552 \pm 23 ; \mathrm{P}<0.05)$ (Fig. 4A and B). However, treatment with 3-MA or chloroquine did not change the MFI of Notch1 $(560 \pm 39$ and $570 \pm 30$, respectively) compared to that in the control group. Furthermore, western blot analysis showed that treatment with autophagy inducer rapamycin reduced the expression of Notch1 and Hes1, whereas 3-MA and chloroquine did not affected the expression of Notch1, but both increased Hes1 expression levels (Fig. 4C and D).

\section{Discussion}

Transdifferentiation of BMSCs into neurons provides a novel approach for the treatment of neurological diseases by cell transplantation $(9,10)$. However, in vitro, the efficacy of BMSC proliferation and differentiation is low and the function of differentiated mature cells is also limited; furthermore, the underlying mechanisms of these processes have largely remained elusive (11-13). Autophagy is a process during which cells digest their own long-lived proteins and organelles under conditions of nutritional starvation and stress (14-16). Autophagy has an important role in cell proliferation, differentiation and apoptosis (17-20), and can rapidly and efficiently degrade transcription factors, enzymes, adhesion molecules and certain secreted proteins $(21,22)$, which are important for stem-cell proliferation and differentiation (23-25). Further studies focusing on autophagy in BMSCs may reveal the underlying mechanisms of proliferation and differentiation, and thereby provide potential strategies for clinical treatment.
The LC3 protein is localized in the membrane surface of autophagosomes. Upon the induction of autophagy, LC3-I binds to phosphatidylethanolamine on the membrane surface of the autophagosome and is then modulated by autophagy-related 7 (Atg7) to form LC3-II. As the levels of LC3-II are positively correlated with the number of autophagic vacuoles, it is considered to be a marker for autophagy (26). P62 is able to bind with LC3 and targeting proteins, which are then degraded in lysosomes. P62 is extensively degraded when the activity of autophagy is increased, while it accumulates in the cytoplasm when autophagy is attenuated, suggesting that P62 levels reflect the level of autophagic activity (27). Mammalian target of rapamycin (mTOR) and the phosphoinositide-3 kinase (PI3K)/AKT pathway are the most important signaling pathways involved in the regulation of autophagy (28). Rapamycin can enhance autophagic activity by inhibiting the mTOR signaling pathway (28), which explains for the result of the present study that rapamycin treatment enhanced LC3-II levels and reduced P62 levels in BMSCs. 3-MA inhibits autophagy by decreasing the activity of type III PI3K signaling (29). Thus, treatment of BMSCs with 3-MA reduced the expression of LC3-II and enhanced P62 in the present study. Chloroquine is able to inhibit autophagy at the late stages by preventing the fusion of autophagosomes and lysosomes (30). Thus, in the present study, treatment with chloroquine slightly increased LC3-II, while it markedly enhanced the expression of P62 in BMSCs.

Deficiency of Atg7 in hematopoietic stem cells has been reported to promote the amplification of bone marrow precursor cells, thus inducing invasive bone marrow hyperplasia and presenting the phenotype of acute myeloid leukemia (25). Autophagy has been suggested to occur in BMSCs $(31,32)$, and is enhanced under stress conditions, such as hypoxia (33). However, the role of autophagy in BMSC apoptosis has remained to be elucidated. Autophagy has been shown to exert cytoprotective effects under stress conditions $(34,35)$. Hypoxia and starvation are known to promote autophagy and decrease apoptosis in BMSC, while 3-MA treatment increases BMSC apoptosis (36). Ugland et al (37) showed that autophagy can be activated via the cyclic adenosine monophosphate (cAMP) signaling pathway in BMSCs, and that the activated cAMP is able to inhibit cell proliferation though modulation of extracellular signal-regulated kinase-mediated activation of cyclin E, thus recruiting autophagy-associated protein Beclin1 to surround the nucleus, which resulted in the formation of autophagic bodies.

In the present study, rapamycin was used to induce autophagy in BMSCs, which was demonstrated to decrease the S-phase population of the cell cycle and promote apoptosis, while treatment with 3-MA and chloroquine exerted the opposite effects, suggesting that activation of autophagy inhibits BMSC proliferation. Autophagy may be a double-edged sword, which can have pro-survival effects under stress conditions to promote cell growth and proliferation, while inhibiting cell proliferation and promoting apoptosis upon excessive activation by degrading proteins and organelles essential for cell proliferation (38).

The present study revealed that rapamycin-induced autophagy enhanced the expression of neuron-specific markers NSE and MAP2. These findings suggested that autophagy is 
involved in BMSC differentiation. Zeng and Zhou (2) found that autophagy is enhanced during the process of retinoic acid-induced differentiation of N2a neuroblastoma cells. Furthermore, the differentiation ability was inhibited by treatment with autophagy inhibitors 3-MA and LY294002 and by RNA interference-mediated suppression of Beclin1. In addition, the mTOR pathway was shown to be inhibited during N2a neuroblastoma cell differentiation, and treatment with mTOR inhibitor rapamycin promoted neurite extension, increased the cell size and enhanced the expression of neuron-specific markers (1). Furthermore, deficiency of Atg7 and Atg5 as well as treatment with 3-MA has been shown to inhibit BMSC differentiation into adipocytes, suggesting that autophagy has an important role in BMSC differentiation into adipocytes (39). Li et al (40) have shown that autophagy is activated during the process of BMSC differentiation into neurons, and that treatment with rapamycin increased the expression levels of Tau and MAP2, while 3-MA had the opposite effect.

The present study provided evidence that the degradation of 'stemness'-associated proteins and the production of neuron-specific proteins is part of the process of BMSC differentiation into neurons. Induction of autophagy was indicated to accelerate this process and thus represents an approach for stimulating cell differentiation, increasing the expression of neuron-specific markers, and promoting BMSC differentiation into neurons.

Notch, a regulatory factor and an important signaling pathway controlling cell fate, has an important role in growth, development, proliferation, differentiation and apoptosis of various cell types (41-43). A previous study by our group demonstrated that the expression of Notch1 and Jag1 genes and their downstream targeted genes PS1 and Hes1 were significantly reduced during the process of human mesenchymal stem cell differentiation into neurons in vitro (44), suggesting that Notch1 is inhibited during the process of neuronal differentiation. Thus, the Notch1 signaling pathway is considered an important regulator for BMSC differentiation into neurons (4).

The present study examined the association between autophagy and the Notch1 signaling pathway in BMSCs and found that induction of autophagy inhibits Notch1. This affects the ability of BMSCs to differentiate into neurons, which is supported by the evidence that the Notch1 signaling pathway and autophagy are linked (45-47). Thus, autophagy may inhibit proliferation and promote differentiation by inhibiting the Notch1 signaling pathway in BMSCs.

The results of the present study demonstrated that induction of autophagy decreased the S-phase population and promoted neuronal differentiation of BMSCs, while inhibition of autophagy reduced apoptosis and neuronal differentiation. These processed were regulated by modulation of the Notch1 signaling pathway. The present study suggested that autophagy has an important role in BMSC proliferation and differentiation. Further study is required to clarify the underlying mechanisms of BMSCs differentiation in vivo.

\section{Acknowledgements}

The present study was supported by National Nature Science Foundation of China (grant nos. 81171250 and 31100790).

\section{References}

1. Lapierre LR, Gelino S, Meléndez A and Hansen M: Autophagy and lipid metabolism coordinately modulate life span in germline-less C. elegans. Curr Biol 21: 1507-1514, 2011.

2. Zeng M and Zhou JN: Roles of autophagy and mTOR signaling in neuronal differentiation of mouse neuroblastoma cells. Cell Signal 20: 659-665, 2008.

3. Greenwald I and Kovall R: Notch signaling: Genetics and structure. WormBook 17: 1-28, 2013.

4. Chiba S: Notch signaling in stem cell systems. Stem cells 24: 2437-2447, 2006.

5. Bray SJ: Notch signalling: A simple pathway becomes complex. Nat Rev Mol Cell Biol 7: 678-689, 2006. ;

6. Louvi A and Artavanis-Tsakonas S: Notch and disease: A growing field. Semin Cell Dev Biol 23: 473-480, 2012.

7. Chen BY, Wang X, Chen LW and Luo ZJ: Molecular targeting regulation of proliferation and differentiation of the bone marrow-derived mesenchymal stem cells or mesenchymal stromal cells. Curr Drug Targets 13: 561-571, 2012.

8. Woodbury D, Schwarz EJ, Prockop DJ and Black IB: Adult rat and human bone marrow stromal cells differentiate into neurons. J Neurosci Res 61: 364-370, 2000.

9. Zhang R, Li J and Xie J: Efficient in vitro labeling rabbit bone marrow-derived mesenchymal stem cells with SPIO and differentiating into neural-like cells. Mol Cells 37: 650-655, 2014.

10. Caplan AI: Why are MSCs therapeutic? New data: New insight. J Pathol 217: 318-324, 2009.

11. Anbari F, Khalili MA, Bahrami AR, Khoradmehr A, Sadeghian F, Fesahat $\mathrm{F}$ and Nabi A: Intravenous transplantation of bone marrow mesenchymal stem cells promotes neural regeneration after traumatic brain injury. Neural Regen Res 9: 919-923, 2014.

12. Jing L, Jia Y, Lu J, Han R, Li J, Wang S, Peng T and Jia Y: MicroRNA-9 promotes differentiation of mouse bone mesenchymal stem cells into neurons by Notch signaling. Neuroreport 22: 206-211, 2011.

13. Hayashi T, Wakao S, Kitada M, Ose T, Watabe H, Kuroda Y, Mitsunaga K, Matsuse D, Shigemoto T, Ito A, et al: Autologous mesenchymal stem cell-derived dopaminergic neurons function in parkinsonian macaques. J Clin Invest 123: 272-284, 2013.

14. Todd LR, Gomathinayagam R and Sankar U: A novel Gfer-Drp1 link in preserving mitochondrial dynamics and function in pluripotent stem cells. Autophagy 6: 821-822, 2010.

15. Mukhopadhyay S, Panda PK, Sinha N, Das DN and Bhutia SK: Autophagy and apoptosis: Where do they meet? Apoptosis 19: 555-566, 2014.

16. Meléndez A and Neufeld TP: The cell biology of autophagy in metazoans: A developing story. Development 135: 2347-2360, 2008.

17. Mizushima N, Levine B, Cuervo AM and Klionsky DJ: Autophagy fights disease through cellular self-digestion. Nature 451: 1069-1075, 2008.

18. Yang Z and Klionsky DJ: Mammalian autophagy: Core molecular machinery and signaling regulation. Curr Opin Cell Biol 22: 124-131, 2010.

19. Yorimitsu T and Klionsky DJ: Autophagy: Molecular machinery for self-eating. Cell Death Differ 12 (Suppl 2): S1542-S1552, 2005.

20. Guan JL, Simon AK, Prescott M, Menendez JA, Liu F, Wang F, Wang C, Wolvetang E, Vazquez-Martin A and Zhang J: Autophagy in stem cells. Autophagy 9: 830-849, 2013.

21. Zirin J and Perrimon N: Drosophila as a model system to study autophagy. Semin Immunopathol 32: 363-372, 2010.

22. Rubinsztein DC, Gestwicki JE, Murphy LO and Klionsky DJ: Potential therapeutic applications of autophagy. Nat Rev Drug Discov 6: 304-312, 2007.

23. Coller HA, Sang L and Roberts JM: A new description of cellular quiescence. PLoS Biol 4: e83, 2006.

24. Phadwal K, Watson AS and Simon AK: Tightrope act: Autophagy in stem cell renewal, differentiation, proliferation and aging. Cell Mol Life Sci 70: 89-103, 2013.

25. Mortensen M, Watson AS and Simon AK: Lack of autophagy in the hematopoietic system leads to loss of hematopoietic stem cell function and dysregulated myeloid proliferation. Autophagy 7: 1069-1070, 2011

26. Kabeya Y, Mizushima N, Yamamoto A, Oshitani-Okamoto S, Ohsumi Y and Yoshimori T: LC3, GABARAP and GATE16 localize to autophagosomal membrane depending on form-II formation. J Cell Sci 117: 2805-2812, 2004. 
27. Komatsu M, Kurokawa H, Waguri S, Taguchi K, Kobayashi A Ichimura Y, Sou YS, Ueno I, Sakamoto A, Tong KI, et al: The selective autophagy substrate p62 activates the stress responsive transcription factor Nrf2 through inactivation of Keap1. Nat Cell Biol 12: 213-223, 2010.

28. Martelli AM, Evangelisti C, Chiarini F, Grimaldi C, Cappellini A, Ognibene A and McCubrey JA: The emerging role of the phosphatidylinositol 3-kinase/Akt/mammalian target of rapamycin signaling network in normal myelopoiesis and leukemogenesis. Biochim Biophys Acta 1803: 991-1002, 2010.

29. Wu YT, Tan HL, Shui G, Bauvy C, Huang Q, Wenk MR, Ong CN, Codogno P and Shen HM: Dual role of 3-methyladenine in modulation of autophagy via different temporal patterns of inhibition on class I and III phosphoinositide 3-kinase. J Biol Chem 285: 10850-10861, 2010.

30. Yoon YH, Cho KS, Hwang JJ, Lee SJ, Choi JA and Koh JY: Induction of lysosomal dilatation, arrested autophagy and cell death by chloroquine in cultured ARPE-19 cells. Invest Ophthalmol Vis Sci 51: 6030-6037, 2010.

31. Pantovic A, Krstic A, Janjetovic K, Kocic J, Harhaji-Trajkovic L, Bugarski D and Trajkovic V: Coordinated time-dependent modulation of AMPK/Akt/mTOR signaling and autophagy controls osteogenic differentiation of human mesenchymal stem cells. Bone 52: 524-531, 2013.

32. Lee Y, Jung J, Cho KJ, Lee SK, Park JW, Oh IH and Kim GJ: Increased SCF/c-kit by hypoxia promotes autophagy of human placental chorionic plate-derived mesenchymal stem cells via regulating the phosphorylation of mTOR. J Cell Biochem 114: 79-88, 2013.

33. Wu J, Niu J, Li X, Li Y, Wang X, Lin J and Zhang F: Hypoxia induces autophagy of bone marrow-derived mesenchymal stem cells via activation of ERK1/2. Cell Physiol Biochem 33: $1467-1474,2014$

34. Li N, Zhang Q, Qian HY, Jin C, Yang Y and Gao R: Atorvastatin induces autophagy of mesenchymal stem cells under hypoxia and serum deprivation conditions by activating the mitogen-activated protein kinase/extracellular signal-regulated kinase pathway. Chin Med J (Engl) 127: 1046-1051, 2014.

35. Song $\mathrm{C}$, Song $\mathrm{C}$ and Tong F: Autophagy induction is a survival response against oxidative stress in bone marrow-derived mesenchymal stromal cells. Cytotherapy 26: 1361-1370, 2014.
36. Wang L, Hu X, Zhu W, Jiang Z, Zhou Y, Chen P and Wang J: Increased leptin by hypoxic-preconditioning promotes autophagy of mesenchymal stem cells and protects them from apoptosis. Sci China Life Sci 57: 171-180, 2014.

37. Ugland H, Naderi S, Brech A, Collas P and Blomhoff HK: CAMP induces autophagy via a novel pathway involving ERK, cyclin E and Beclin 1. Autophagy 7: 1199-1211, 2011.

38. Shintani T and Klionsky DJ: Autophagy in health and disease: A double-edged sword. Science 306: 990-995, 2004.

39. Singh R, Xiang Y, Wang Y, Baikati K, Cuervo AM, Luu YK, Tang Y, Pessin JE, Schwartz GJ and Czaja MJ: Autophagy regulates adipose mass and differentiation in mice. J Clin Invest 119: 3329-3339, 2009.

40. Li Y, Wang C, Zhang G, Wang X, Duan R, Gao H, Peng T, Teng J and Jia Y: Role of autophagy and mTOR signaling in neural differentiation of bone marrow mesenchymal stem cells. Cell Biol Int 38: 1337-1343, 2014.

41. Patel PN, Yu XM, Jaskula-Sztul R and Chen H: Hesperetin activates the Notch1 signaling cascade, causes apoptosis, and induces cellular differentiation in anaplastic thyroid cancer. Ann Surg Oncol 21 Suppl 4: S497-S504, 2014.

42. Su BH, Qu J, Song M, Huang XY, Hu XM, Xie J, Zhao Y, Ding LC, She L, Chen J, et al: NOTCH1 signaling contributes to cell growth, anti-apoptosis and metastasis in salivary adenoid cystic carcinoma. Oncotarget 5: 6885-6895, 2014.

43. Zhao C, Guo H, Li J, Myint T, Pittman W, Yang L, Zhong W, Schwartz RJ, Schwarz JJ, et al: Numb family proteins are essential for cardiac morphogenesis and progenitor differentiation. Development 141: 281-295, 2014

44. Xing Y, Bai RY, Yan WH, Han XF, Duan P, Xu Y and Fan ZG: Expression changes of Notch-related genes during the differentiation of human mesenchymal stem cells into neurons. Sheng Li Xue Bao 59: 267-272, 2007 (In Chinese).

45. Barth JM and Köhler K: How to take autophagy and endocytosis up a notch. Biomed Res Int 2014: 960803, 2014.

46. Kwon MH, Callaway H, Zhong J and Yedvobnick B: A targeted genetic modifier screen links the SWI2/SNF2 protein domino to growth and autophagy genes in Drosophila melanogaster. G3 (Bethesda) 3: 815-825, 2013.

47. Yamamoto S, Charng WL and Bellen HJ: Endocytosis and intracellular trafficking of Notch and its ligands. Curr Top Dev Biol 92: 165-200, 2010. 